\title{
Progress of Solid-Phase Microextraction Coatings and Coating Techniques
}

\author{
Guibin Jiang*, Minjia Huang, Yaqi Cai, Jianxia Lv, and Zongshan Zhao \\ State Key Laboratory of Environmental Chemistry and Ecotoxicology, Research Center for Eco-Environmental Sciences, Chinese Academy of \\ Sciences, P.O. Box 2871, Beijing 100085, China.
}

\begin{abstract}
Solid-phase microextraction (SPME) has been popular as an environmentally friendly sample pretreatment technique to extract a very wide range of analytes. This is partly owing to the development of SPME coatings. One of the key factors affecting the extraction performances, such as the sensitivity, selectivity, and reproducibility, is the properties of the coatings on SPME fibers. This paper classifies the materials used as SPME coatings and introduces some common preparation techniques of SPME coating in detail, such as sol-gel technique, electrochemical polymerization technique, particle direct pasting technique, restricted access matrix SPME technique, and molecularly imprinted SPME technique.
\end{abstract}

\section{Introduction}

Solid-phase microextraction (SPME) is a sample preparation technique developed by Pawliszyn et al. (1,2). The process of SPME includes two major operation steps: extraction and desorption. Because both extraction and desorption take place on the coatings, the properties of the coatings determine the performance of SPME. Based on this fact, the development of new coatings has been in great demand for a long time. In recent years, the quantity of papers discussing new coatings for SPME has increased rapidly. In this paper, many of the present coatings are classified, and the common preparation techniques are described in detail.

\section{Discussion}

\section{The classifying of coatings}

In the book Applications of Solid-Phase Microextraction, Górecki (3) sorted the coatings from Supelco (Bellefonte, PA) into two different categories: high-viscosity liquid coatings and solid coatings. He also described the extraction mechanism for both

\footnotetext{
* Author to whom correspondence should be addressed: email gbjiang@mail.rcees.ac.cn.
}

kinds of coatings, respectively. For liquid coatings, the extraction of analytes is obtained by the absorption, in which the analytes can transfer from the surface of coatings to the inside. This kind of coating includes the common stationary phases polydimethylsiloxane (PDMS) and polyacrylate (PA). For solid coatings, the extraction of analytes is based on adsorption, in which the analytes may only stay on the surface of the coatings. Some composite coatings such as PDMS-divinylbenzene (DVB), carbowax-DVB, carbowax-templete resin, etc. from Supelco are solid coatings with porous surfaces. They can only extract analytes by adsorption. During the extraction process, the mechanism of adsorption is much more complex than absorption.

With the development of the SPME technique, more and more new materials are utilized in the preparation of coatings. More than half of currently available coatings are solid coatings. However, they are divided by the properties of the materials; the solid coatings are multifarious. Based on organic and inorganic types, there are two primary kinds of coatings: organic and inorganic.

\section{Organic coating}

Organic polymer materials are the first kind of material used as SPME coatings. Because traditional SPME fibers utilize fusedsilica fiber as solid-phase carriers, compared with inorganic materials, organic polymer materials are easier to affix with fiber. In addition, at high temperature, the thermal coefficient of expansions for polymer materials and fused-silica fibers are similar, so it is not easy for the coating to flake off from fibers. Furthermore, a great diversity of structures is another important reason why polymers are very popular.

The analytes extracted on the fiber coating with SPME can be expressed by equation 1 . Based on equation 1 , for analytes with high $K_{f s}$ values, the amount of extracted analytes $(n)$ is greater than that for those analytes that have a low $K_{f s}$. In order to increase the $K_{f s}$ value, some organic polymers containing particular functional groups were chosen to increase the interaction between polymers and coatings (4). For example, if the analytes contain $-\mathrm{OH}$ or $-\mathrm{NH}_{2}$, the polymer, which includes the groups (such as $-\mathrm{OH}$ or $-\mathrm{NH}_{2}$ ) that can interact with the group of analytes by hydrogen bond, can be selected. 
$n=K_{f s} V_{f} V_{s} C_{o} / K_{f s} V_{f}+V_{s}$

Eq. 1

where $V_{f}$ is the volume of the fiber and $V_{s}$ is the volume of the sample.

Therefore, several kinds of polymers with different functional groups have been synthesized and obtained. Descriptions of the categories of these organic coatings follow.

\section{Coatings based on organosiloxane materials}

Polysiloxane is a significant SPME coating material because of its high thermal stability, good film-forming performance, and extensive use as stationary phase for gas chromatography (GC). Also, it will be easier to introduce various functional groups to the side chains than other materials (5). Researchers in the department of Chemistry at Wuhan University have done plenty of work in this field. The group of $\mathrm{Wu}(6)$ had prepared a nonpolar coating, polymethylvinylsiloxane (PMVS), which has strong extraction capacity toward nonpolar organic contamination. In the application of extracting benzene derivatives, such as ethylbenzene, $o$-xylene, and $m$-xylene from an aqueous phase, PMVS coating showed better extraction capacity than $100 \mu \mathrm{m}$ PDMS coating. By using the sol-gel technique, they bonded polyethylene glycols to the backbone of PDMS and successfully extracted benzene derivatives, phenols compounds, phthalate esters, polycyclic aromatic hydrocarbons, and organochloride pesticides by the coating. The results of the experiment proved that this new coating, with polar groups, can extract a number of polar and nonpolar analytes (7). The group of Zeng (8) prepared a polymethylphenylvinylsiloxane coating, which showed better extraction capacity than the commercial extraction fibers in the application of extracting benzene derivatives and polycyclic aromatic hydrocarbons. The group of $\mathrm{Wu}(9)$ bonded Fullerene (C60) to the side chain of polysiloxane and applied this coating in extracting benzene derivatives, phthalate esters, and polycyclic aromatic hydrocarbons in water. The result showed that the method had a wide linear range and good sensitivity. They also utilized this fiber in the determination of hydrocarbon compounds and aromatic compounds, though they found that this fiber had a good ability to extract polar compounds (10). In order to produce a more stable structure, they bonded hydroxyls of fullerenes into the skeleton of polysiloxane (Figure 1) by the sol-gel technique and tested the extraction ability of the new coating by extracting polychlorinated biphenyl, polycyclic aromatic hydrocarbon, and aromatic amine, by which they obtained very high sensitivity (11). Zeng et al. (12) also bonded crown ethers to the side chain of polysiloxane to obtain a new coating, which had a very high capacity in extracting phenols compounds. Some crown ethers with other substitutes were also bonded to the side chain of polysiloxane as SPME coatings (Figure 2) (13-18) to extract many kinds of organic compounds, such as organophosphorous pesticides, and showed excellent extraction characteristics for many organic compounds. Recently, the group of Zeng (19) also bonded calyx[4]arenes to the side chain of polysiloxane (Figure 3), which has a good ability to extract a supermolecule, and it also has good selectivity for polar aromatic amine compounds. In addition, they bonded calixarenes in order to extract polar compounds $(20,21)$.

Gongke Li et al. (22) bonded the OH-group of $\gamma$-cyclodextrin to the terminal of polysiloxane, pasted it on the surface of a fused-

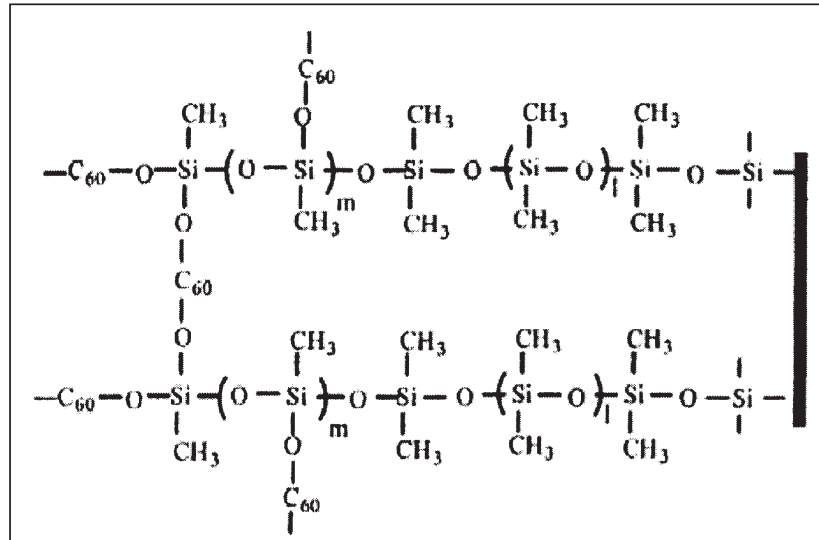

Figure 1. The structure of bonded fullerene polysiloxane stationary phase as an SPME coating.

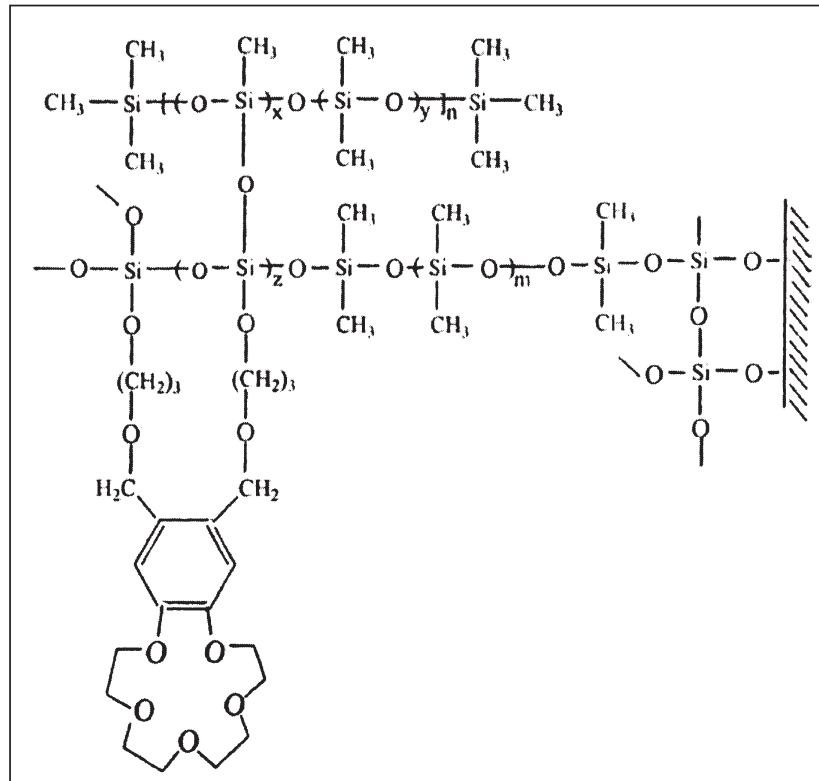

Figure 2. The structure of bonded crown ethers polysiloxane stationary phase as an SPME coating.

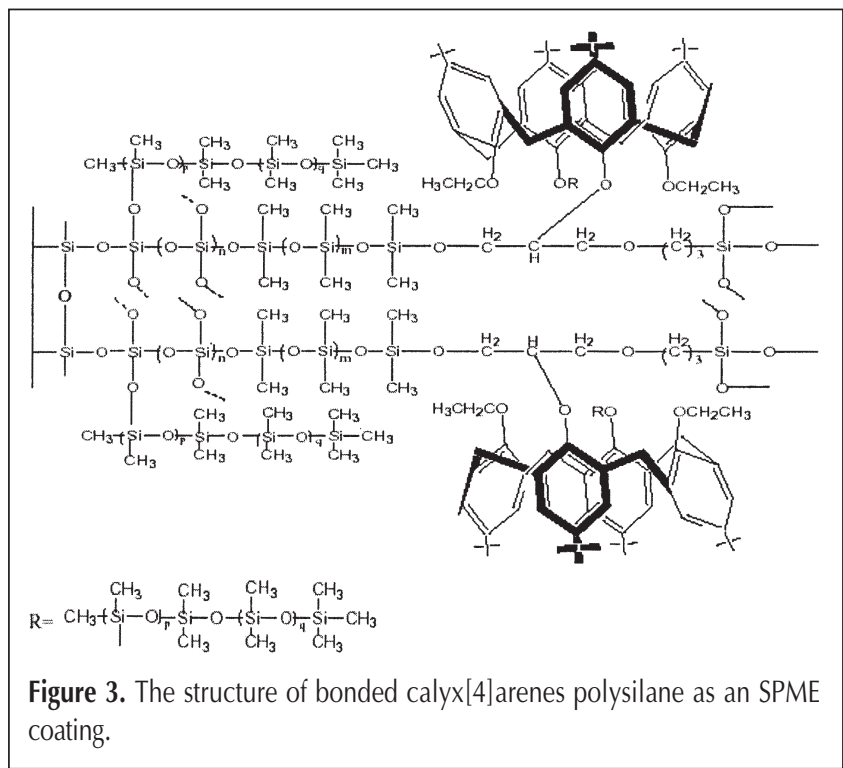


silica fiber by high-temperature epoxy, and used it as an SPME coating. Then, they utilized microwave-assisted extraction and detected phenol compounds in water. Figure 4 is the schematic of the synthesizing process of this kind of cyclodextrin. They also prepared a poly(dimethylsiloxane)- $\beta$-cyclodextrin SPME membrane for the determination of polycyclic aromatic hydrocarbons and phenolic compounds in the water samples (23). The advantages of the method seemed to be high extraction effect and low cost.

Bonded mesoporous silica material also have been applied in the SPME field. Recently, Du et al. (24) coated a stainless steel wire with phenyl-functionalized MCM-41-type mesoporous silica materials and used this wire as the SPME fiber for the extraction of aromatic compounds. The results showed that this novel coating exhibited many advantages, such as high sensitivity and selectivity, because of its large surface area and small mesopores, high mechanical strength, good thermal stability, easy preparation, and long lifetime. Fan et al. (25) introduced the ordered mesoporous silica modified with octadecyl groups into the capillary for in-tube SPME, used the in-tube SPME-high-performance liquid chromatography (HPLC) system, and determined bisphenol A in water samples.

\section{Coatings based on polyamine materials (polyamide and polyimide)}

The polyamine materials have strong polarity, high thermal stability, and good wetting ability on the surface of the silica fiber. Thus, they are often used as a protection coating for GC capillary columns. Whang et al. (26) detected $\mathrm{Cr}$ (III) in water using polyimide-coating-containing fibers as SPME fibers, and the result of their experiment exhibited very high sensitivity. Yang et al. (27) successfully extracted dichlorvos in blood sample and morphine and phenobarbital in urine samples by using polyamide SPME membranes containing Tenax and pyridine.

\section{Coatings based on other polymeric materials}

Farajzadeh et al. (28) coated both cellulose acetate and polyvinyl chloride (PVC) on silver wires and used this porous material as an SPME coating to extract alkanes in water samples, and they found that the coating has good selectivity for extracting alkanes with a carbon number higher than 10 , especially for $\mathrm{C}_{12}-\mathrm{C}_{15}$.

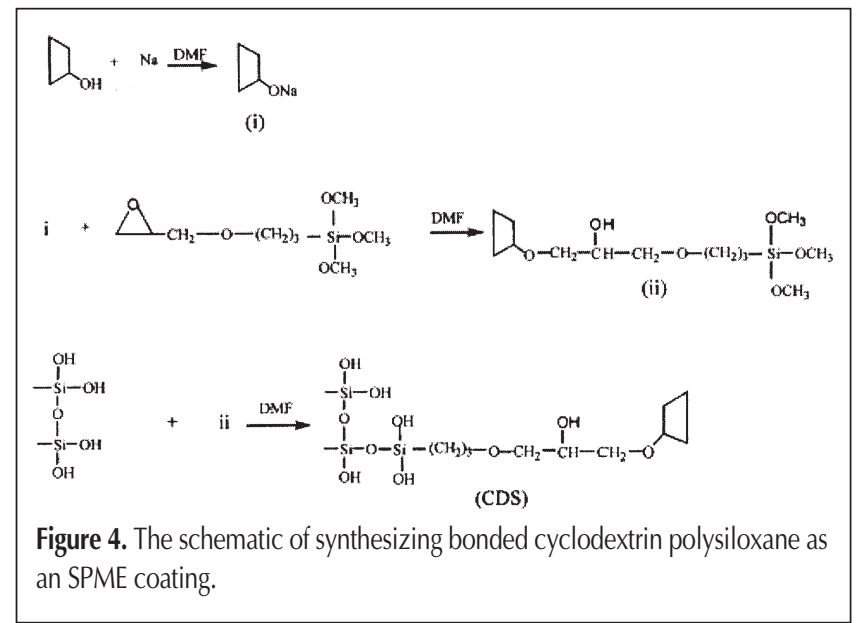

Thompson et al. (29) coated poly-(3-methylthiophene) on the surface of platinum micro-fiber electrodes and utilized the electrodes to successfully extract arsenate ions from aqueous solutions by an electrochemical method without derivatization. This was the first time conductive polymers were used as SPME coating materials. Shortly after, they synthesized polythiophene, which was modified with longer alkyl groups and made for the speciation analysis of arsenic by using an electrochemically aided SPME (EASPME) method and HPLC-inductively coupled plasma-MS technique (30). Afterwards, other conductive polymers were used in SPME. Nyholm et al. (31) prepared two kinds of polypyrrole coatings, which were doped with perchlorate and $p$-toluenesulfonate, respectively, by an electrochemical method. They also designed a self-made EASPME apparatus (Figure 5). The results showed that under a constant potential of $+1.0 \mathrm{~V}$ and $-0.5 \mathrm{~V}$, relative to the calomel electrodes, this apparatus is wellsuited to extract parts per million level anions (like chloride, nitrite, bromide, sulfate, and phosphate) and cations (like calcium, cobalt, and zinc). Kadir et al. (32,33) found that SPME coatings based on overoxidized sulfonated polypyrrole (OSPPY) materials can be used to extract a great variety of cationic analytes, including metallic ions in elctrochemically controlled SPME. Bagheri et al. (34) prepared an aniline-based coating to extract phenols from water by the electropolymerization method.

The research group of Pawliszyn (35) prepared three kinds of different doping polypyrroles: perchlorate, chloride, and poly (styrensulfonate-pyrrole), and they concentrated sodium methy-

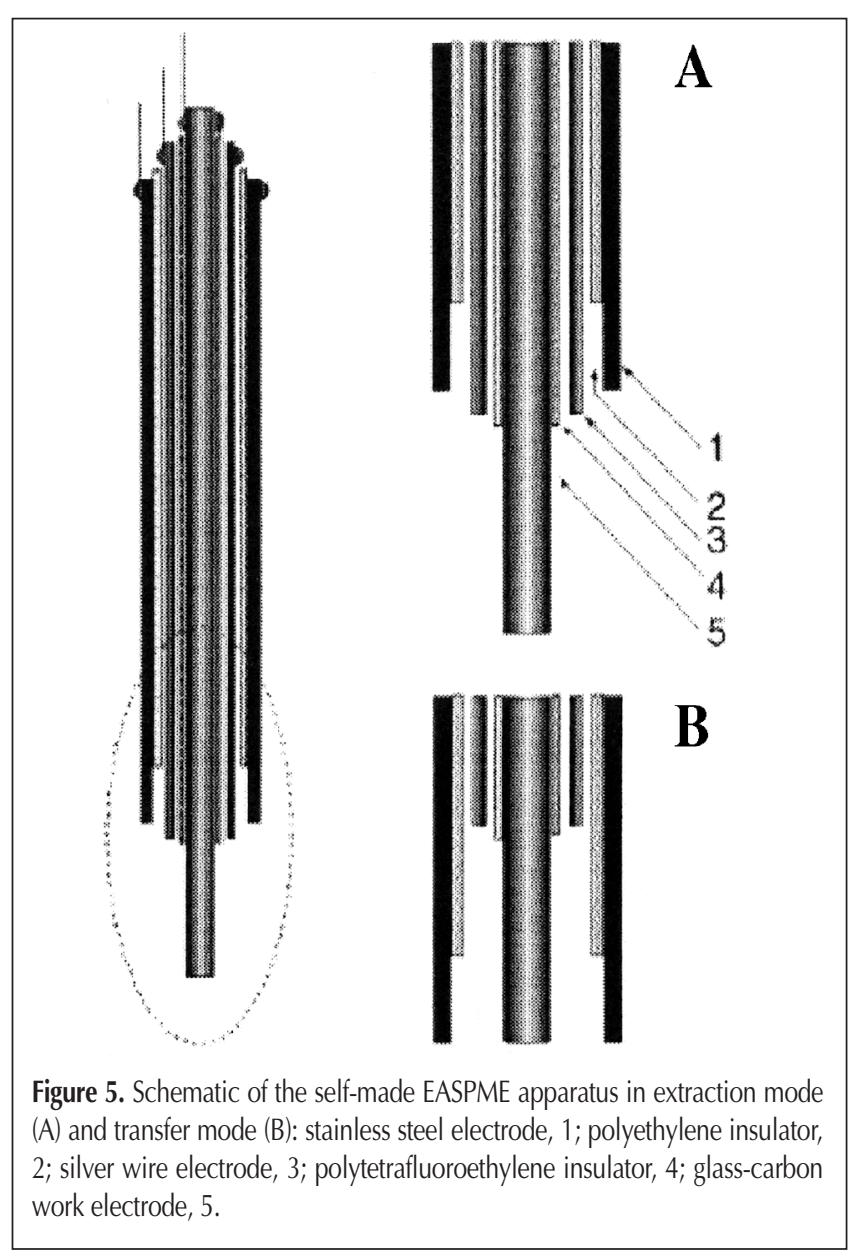


larsonates, glutamate, perchlorate, and dopamine by three different polypyrroles, respectively, then desorbed the compounds to water by electrolysis and injected them into the HPLC-MS directly by flow injection analysis. They also prepared some polypyrrole with the organic synthesis method and electrochemical polymerization method to extract several analytes. Most of their work was focused on coating polypyrrole inside of the capillaries and using the in-tube SPME method. Some of their work dealt with fiber SPME that extracted volatile organic compounds by fiber with a polypyrrole coating (36-44). In another paper, they coated polypyrrole on the surface of a fused-silica fiber and utilized surface-enhanced laser desorption/ionization, combined with MS, to analyze small-molecule peptides (45). Alizadeh et al. (46) used an electrochemical polymerization method to coat dodecylsulfate-doped polypyrrole on a platinum wire and extracted polycyclic aromatic hydrocarbons by a headspace (HS)-SPME method. Then, they used GC-flame ionization detection (FID) to analyze the compound with the sub-parts per billion detection limits. Tamer (33) synthesized overoxidized sulfonated polypyrrole using an electrochemical method and applied the polymer for the selective extraction of trace levels of nickel and cadmium ions.

In 2003, the research group of Pawliszyn (47) utilized polypyrrole fibers to study in vivo pharmacokinetics in dog blood. It was a great breakthrough for the application of in vivo SPME. They coated polypyrrole on the surface of metal wires by the electrochemical polymerization method. The excellent strength of the metal wires provided the possibility to do experiments in vivo. The polypyrrole SPME fibers were placed (through a catheter) into the cephalic vein of the lower front leg and then analyzed the samples by LC-MS-MS. The movement of the blood was expected to increase the extraction ability in a predetermined time.

In recent years, molecularly-imprinted polymers have been used in many analytical chemistry research fields, such as chromatographic stationary phases, selective materials for biosensors, and solid-phase adsorbents. Both Koster et al. (48) and Pawliszyn et al. (49) have done a lot work on the application of molecularlyimprinted polymers on SPME coating. They coated several methacrylate polymers, which had been synthesized with imprinted $\beta$-blockers, directly onto the surfaces of the silica fiber and used them as an SPME fiber for the extraction and determination of $\beta$-blockers in biological fluids. The molecularlyimprinted polymer selectivity simplified the sample preparation technique and chromatographic separation.

\section{Inorganic coatings}

For SPME coating materials, adsorptive capacity is an important factor. Although inorganic compounds are not as diversified as organic coatings as far as structures are concerned, some inorganic compounds with high adsorptive capacity are also popular as SPME coatings.

\section{Coatings based on graphite materials}

Activated carbon is a common kind of adsorbent because of its strong adsorptive capacity. Mangani et al. (50) coated graphitized carbon black on the surface of fused-silica fiber and used a selfmodified SPME device to extract volatile organic compounds from water and organochloride from blood and urine samples. The limit of detection of the method was at the approximately 1$\mathrm{pg} / \mathrm{mL}$ level. Djozan et al. (51) had affixed extra fine, porous-layer activated-charcoal powder to SPME fibers by PDMS as adherent and used it to extract benzene derivatives in water. They also inserted pencil lead into SPME fiber tubes and used them as coating directly (52). Jinping Jia et al. (53) explored the possibility of using activated carbon fiber as SPME fibers. Their experiment showed that, as an SPME fiber, the activated carbon fiber had many advantages, such as better resistance to organic solvents, better endurance to high temperature, longer lifetime, and a good extractability for chlorinated hydrocarbons.

Although graphite and activated carbon have strong adsorptive capacity, their selectivity for analytes is poor. However, being electrode material, glassy carbon is quite different from graphite because the arrays of carbon atoms in different layers are not accurately orientated, and the flat molecular surface provides special selectivity and retaining ability. Burk et al. (54) used polycrystalline graphite to extract nonionic surfactants, such as Triton X-100, and obtained the similar selectivity as commercial PDMS-DVB and carbowax-template resin. Olesik et al. (55) coated a low-temperature glassy carbon (LTGC) membrane, which can endure approximately $1000^{\circ} \mathrm{C}$ on stainless steel wires. The LTGC-coated silica particles were also immobilized onto stainless steel wire fibers and followed by GC-FID analysis. The result of experiments indicated that this membrane had good selectivity to the polar molecules or the molecules with the crosssectional surface. They also detected three peculiar taste and odor compounds in aqueous samples by this method as. The same authors (56) had prepared the same coating on a $4-\mathrm{cm}$ long fiber, and the extraction capability of the fiber was increased greatly. In 2004, they synthesized a fluorinated low-temperature glassy carbon film (57). Compared with many commercial fiber coatings, this kind of fluorinated LTGC membrane has better concentration capability in the application of extraction of the halogen-containing compounds (such as halogenated benzenes).

\section{Coating materials based on metallic compounds}

The solid supports of coating materials based on metallic compounds are mostly metal wires. Metal wires are dipped in solution, and metallic compounds are obtained by chemical or electrochemical methods during the preparation process. For example, Djozan et al. (58) dipped clean copper wire in a solution of $\mathrm{CuCl}_{2}$ to produce a layer of $\mathrm{CuCl}$ microcrystals on the surface of copper wire. This kind of coating could be used to extract volatile amines, and the detection limit of the method was $0.1-0.3 \mathrm{ng} / \mathrm{mL}$. Similar methods were used to prepare anodized aluminum wire (59), electrochemical anodized zinc wire (60), and copper sulfide wire (61). Farajzadeh et al. (62) had prepared three kinds of copper (I) compounds on the surface of copper wire, including copper (I) chloride, copper (I) oxide, and copper (I) sulfide by using the electrochemical method. The experiment result proved that copper (I) chloride exhibited the best extraction effect for amines among the three copper (I) compound coatings, and its detection limit was similar to that obtained with the $\mathrm{CuCl}_{2}$ coating by Djozan et al. (58). They also utilized the special affinity between alumina and hydroxyl groups to extract alcohol compounds with the coating, which was made by the mixture coating of both alumina 
powder and PVC (97:3) on the silver wire. The extraction effect was excellent, and the detection limit was $0.1 \mathrm{ng} / \mathrm{mL}$ (63). The group of $\mathrm{Ou}(64)$ also coated $\gamma-\mathrm{Al}_{2} \mathrm{O}_{3}$ on the surface of fused-silica fiber and successfully detected some volatile organic compounds in indoor air. Oliveira et al. (65) and Silveira et al. (66) coated $\mathrm{Nb}_{2} \mathrm{O}_{5}$ on fiberglass to extract phenols, and they also coated $\mathrm{Nb}_{2} \mathrm{O}_{5}$ on thin glass-ceramic rods to extract alcohols and phenols by HS-SPME. The result showed that the niobium (V) oxide coating had good extraction ability for phenols and alcohols.

\section{Preparation technique of coatings}

For different coating materials and different solid support phases, the preparation techniques are different. The following are some common coating methods.

\section{Coating materials by direct-pasting technique}

A simple device is enough for this technique. For a small amount of production, researchers only need a container and solution. They can dip one end of the silica fiber or metal fiber into the solution for a predetermined time and then cure the coating by UV light or heat. The group of Wu (41) had coated PMVS and $1 \%(\mathrm{~m} / \mathrm{m})$ photosensitizer onto silica fibers by the described method. Olesik et al. (55) designed a smart coating device (Figure 6) in order to obtain better reproducibility of preparation technique.

The described technique is not suitable for lot production. Mani (67) introduced the production process of commercial fibers by Supelco, in the book, Applications of Solid Phase Microextraction. After the silica fibers were drawn by machine, they were immediately cooled to the ambient temperature and drawn

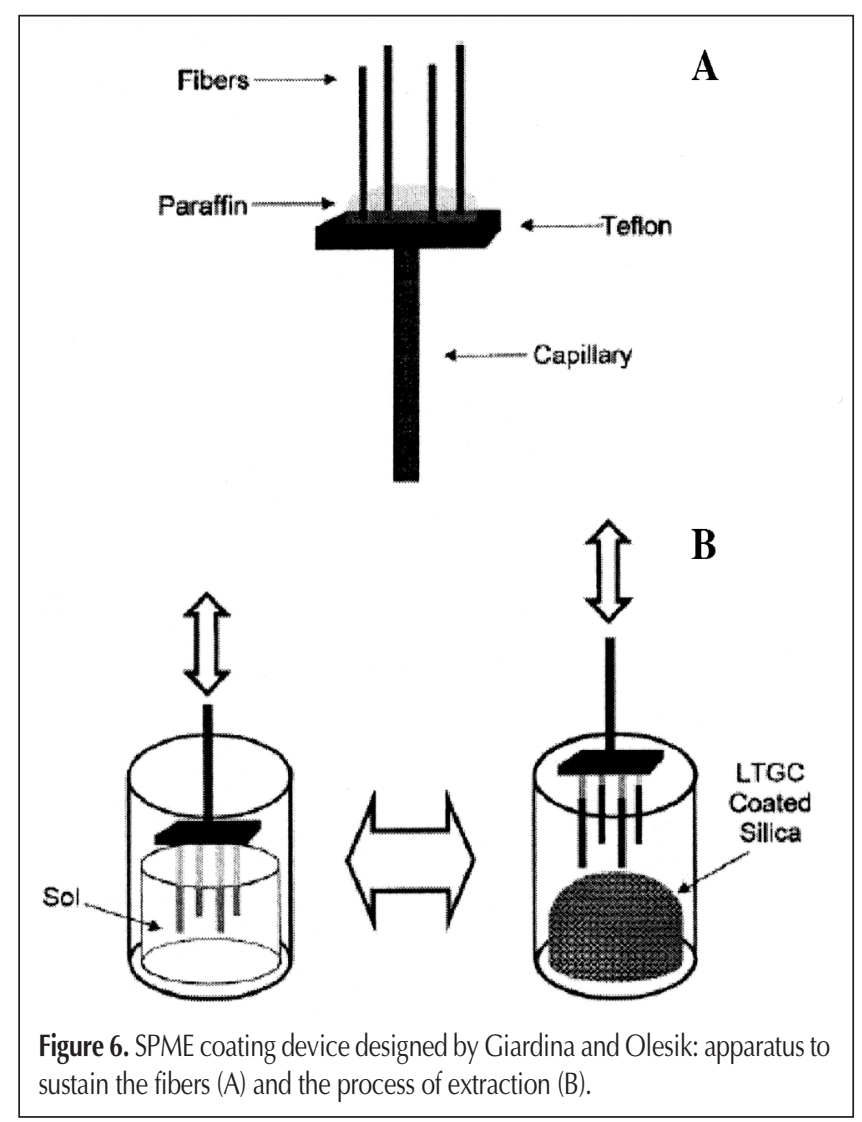

through a container with the coating solution inside (for complex coating, multiple containers are necessary). After the fibers were drawn out of the container, the polymers were coated on the fibers. The fibers were then cured by heat or UV light and then coiled. Two centimeters of the fibers is always enough. It is then cut for the SPME device.

\section{Sol-gel technique}

In 1997, Chong et al. (68) were the first to apply the sol-gel immobilization method technology in the preparation of SPME coatings. The technique can not only make hydroxyl-terminate PDMS bond with the hydroxyls of the surface of silica fibers, but also make hydroxyl-terminate PDMSs bond with each other. Thus, the entire coating formed as net structures and was stabilized with the surface (see Figure 1 for the similar structure). The results of their experiments proved that the maximum temperature of this kind of coating was $100^{\circ} \mathrm{C}$ higher than the normal physical coatings. Both the groups of Wu and Zeng from Wuhan University had prepared many kinds of coating by the sol-gel technique and extracted several kinds of compounds, such as organophosphorus pesticides, organochlorine pesticides, aroma compounds in beer, phenolic compounds, 2-chloroethyl ethyl sulfide, and volatile compounds in red wine (16,69-73). Ligor et al. (74) prepared ethoxy-PDMS-coated fiber and polyurethaneacrylate coatings, which had high thermal stability (in the range of $300^{\circ} \mathrm{C}$ to $450^{\circ} \mathrm{C}$ ). Caruso et al. (75) prepared new sol-gel coating, including the $\mathrm{C} 8$ group bonded on PDMS and applied in HPLC analysis, which had been used to detect organomercury, organoarsenic, organotin, etc., in water with the detection limits of 80-600 $\mu \mathrm{g} / \mathrm{L}$. Recently, Khalid et al. (76) developed a novel zirconia-based hybrid organic-inorganic sol-gel coating and used in-tube SPME to extract some polycyclic aromatic hydrocarbons, aldehydes, and ketones. The experiments showed that the novel sol-gel hybrid zirconia coating had excellent high pH stability and retained the extraction ability even after continuous rinsing with a $0.1 \mathrm{M} \mathrm{NaOH}$ solution for $24 \mathrm{~h}$. Basheer et al. (77) synthesized amphiphilic and hydrophilic oligomers and coated them on fused-silica fibers by a sol-gel technique; they used these fibers to extract many compounds, including organochlorine pesticides, triazine herbicides, estrogens, alkylphenols, and bisphenol-A. These fibers showed excellent SPME performance for the extraction of both polar and nonpolar organic compounds. The sol-gel coating demonstrated long lifetime and high thermal stability up to $280^{\circ} \mathrm{C}$.

Besides the previously described research work using sol-gel synthesis technology, some researchers have developed poly (ethylene glycol)-modified ormosil coating $(78,79)$, poly(ethylenepropyleneglycol) monobutyl ether coating (79), phenyl-functionalized coating (80), and modified ormosil coating fibers for the SPME of many kinds of compounds with very different polarity.

\section{Using shaped materials directly as coatings}

As described earlier, Djozan et al. (52) employed pencil lead as extraction fibers. The pencil lead does not need solid phase carrier support. The research group of Pawliszyn (81) enclosed silica fiber with a Nafion tube to extract polar compounds. Jinping Jia et al. (82) chose suitable reprocessed plant fibers as a matrix to 
obtain fibers in a self-made reactor and then put them in a selfmade SPME holder to extract benzene. They also used activated carbon fiber for the determination of chlorinated hydrocarbons (53). He et al. (83) dipped the terminal of the silica fiber in hydrofluoric acid and found the eroded surface of the fiber had good selectivity for methylmercury. She also detected methylmercury in organisms and sediments by using this fiber combined with the hydride derivatization method. The coatings based on metal materials mentioned previously were all obtained by modifying the metal wires with the organic chemical or electrochemical method. Because of its simple operation, this kind of preparation technique is still popular.

\section{Pasting coatings onto the carriers by adhesives}

This coating technique is a little difficult to operate and it requires an adhesive of good quality. High-temperature epoxy glue was commonly used because of its high thermal stability. Liu et al. $(84,85)$ had affixed C8 and C18 silica particles onto stainless steel wire with high-temperature epoxy glue to extract benzene derivatives, polycyclic aromatic hydrocarbons, and polychlorinated biphenyls. The silica gel particles increased the surface area of coating and, thus, increased the capability of the fiber coating, which is 500 times greater than PDMS coating when extracting the same concentration of benzene derivatives. Djozan et al. (51) prepared a new, porous-layer-activated charcoal coating with PDMS as the adhesive. The group of Pawliszyn (86) proposed a novel, restricted-access SPME method. First, they prepared the SPME fiber by pasting the alkyldiol-silica restricted-access materials onto the steel wire using an epoxy binding agent, and then they interfaced the SPME device with an LC-MS system and developed a method for the determination of some drugs and their metabolites in whole blood. This method exhibited excellent stability. The biggest advantage of this novel method was that there was no requirement to precipitate proteins from the biological sample prior to extraction. By using a similar method, they also prepared cation-exchange restricted-access SPME stainless fiber for the extraction and HPLC determination of angiotensin 1 in the whole blood sample (87). Hou et al. (88) pasted the inorganic-organic mesoporous silica C16-MCM-41 particle onto stainless steel wire by epoxy resin glue, and they used the fiber to determine trace anthracene in environmental samples. The results indicate that by using the fiber coating, the method would exhibit high extraction effect and good selectivity. It was reported that this new material had a very large surface area and high mechanical, chemical, and thermal stability.

Generally speaking, the coating prepared by the pasting technique has a large surface area, and this will result in good extraction capacity. But this technique has its own weakness (e.g., the interaction between coating particles and fiber is not strong enough to stand the solvent from the HPLC mobile phase), so any application of fibers prepared by this technique in HPLC fields should be performend cautiously. In addition, the interference from the adhesive impurities is also a problem to be overcome.

\section{Electrochemical polymerization method}

Some polymers prepared by this method have a good electrical conductivity property. Therefore, this kind of coating is sometimes called conductive polymer, even though in SPME the con- ductivity of the coating sometimes did not matter. The technique has the advantages of easy operation. Moreover, in this method, the strength of metal wires is better than silica fibers; thus, this technique has a wider application range, like in vivo investigation. Besides, because of the great difference of the thermal expansion coefficient between polymer and metal, flaking of polymers may occur at high temperatures if the coatings were prepared with simple physical coating techniques. Polymers prepared by electrochemical polymerization are deposited, point by pont, on the surface of the metal wire. They are too robust to flake off, even at high temperatures.

In the Coatings based on other polymer materials section, most of the polymers coated on metal wires were prepared by this technique. Besides polypyrrole and polythiophene, polyaniline is also a popular conducting polymer. According to Trojanowicz's paper (89), polyaniline had been the most popular conducting polymer in analytical chemistry. It has been used in SPME $(90,91)$, LC $(92,93)$, and ionic chromatography $(94,95)$. Our group $(96,97)$ had electroplated polyaniline onto metal wires by using electrochemical polymerization and detected aromatic amines (aniline, $N, N$-dimethylaniline, $m$-toluidine, 3,4-bichloroaniline, 2-chloroaniline, and 2,4-bimethylaniline) in water with HS-SPME-GC. The linear range was from 4.8 to $27.5 \times 10^{4} \mu \mathrm{g} / \mathrm{L}$, the detection limits were from 0.019 to $1.06 \mu \mathrm{g} / \mathrm{L}$, and the RSDs were $2.02-6.00 \%$ under optimizing condition. The self-made composite polyaniline coating was prepared and aimed at the commercial interface for HPLC and SPME. The fiber was applied to the concentration of three phenol compounds by using direct SPME with HPLC-flourescence detector. The extraction fibers also showed better sensitivity than commercial CW-TPR coatings. Recently, Alireza et al. (98) developed a simple and sensitive method for determinination of anatoxin-a in aqueous samples using SPME-GC-MS. In their method, they prepared the SPME fibers by precipitating polyaniline films directly onto the surface of gold wires using an electrochemical polymerization method. The experiments showed that these polyaniline coated fibers had a good extraction performance for anatoxin-a.

\section{Conclusion}

Generally speaking, more and more novel coatings have been developed, and they were suitable for the analysis of many kinds of compounds with very different properties, such as polarity and volatility. The SPME methods based on these new coating materials have been applied in many analytical fields. In the future, high selectivity, accuracy, sensitivity, long lifetime, and good compatibility with biological system of the SPME coating are still the goal for analysts. Based on a summary of a mass of literature, more efforts should be put on the following aspects in the research of SPME fiber coatings. First, the coating with high selectivity should be paid more attention. Because of their excellent selectivity, the fiber coating materials based on molecularly imprinted polymers and antibodies are absolutely a good choice. Many researchers, such as the group of Pawliszyn have done some excellent work on this aspect, but there is still a huge space in this field for researchers to study. Mullett et al. (99) wrote a review 
paper in which he described some selective SPME coatings, including molecule imprinted polymer (50), bonding antibody technique (100), etc. Moreover, good biological compatibility and in vivo extraction, sampling, and determination by one step is another important trend in analytical chemistry. Thus, the SPME fiber coatings with such properties are definitely in demand; the efforts in this field should be further strengthened. The research on monolith materials is a hot topic in analytical chemistry in recent years, but their application in the SPME field is very limited $(101,102)$. However, we believe that the SPME fiber coatings based on monolith materials should have a bright future because of their unique properties, such as very rapid extraction kinetics, high extraction effect, and good compatibility with biological samples.

\section{References}

1. R.P. Berladi and J. Pawliszyn. The application of chemically modified fused silica fibers in the extraction of organics from water matrix samples and their rapid transfer to capillary columns. Water Pollut. Res. J. Can. 24: 179-91 (1989).

2. C.L. Arthur and J. Pawliszyn. Solid-phase microextraction with thermal desorption using fused silica optical fibers. Anal. Chem. 62: 2145-48 (1990).

3. J. Pawliszyn and T. Górecki. Applications of Solid Phase Microextraction, 1st ed. Royal Society of Chemistry, Corwall, U.K., 1999, pp. 92-108.

4. W.M. Mullett and J. Pawliszyn. The development of selective and biocompatible coatings for solid phase microextraction. J. Sep. Sci. 36: 251-60 (2003).

5. C.Y. Wu and Z.R. Zeng. Modern Capillary Gas Chromatography, 1 st ed. Wuhan University Press, Wuhan, China, 1990, pp. 54-64.

6. D.N. Zhang, C.Y. Wu, and F. Ai. The study of polymer coating in solid phase microextraciton. Chin. J. Chromatogr. 17: 10-13 (1999).

7. Z.Y. Wang, C.H. Xiao, C.Y. Wu, and H.M. Han. High-performance polyethylene glycol-coated solid-phase microextraction fibers using sol-gel technology. J. Chromatogr. A 893: 157-68 (2000).

8. M. Yang, Z.R. Zeng, W.L. Qiu, and Y.L. Wang. Preparation and investigation of polymethylphenylvinylsiloxane-coated solid phase microextraction fibers using sol-gel technology. Chromatographia 56: 73-80 (2002).

9. C.H. Xiao, Z.L. Liu, Z.Y. Wang, C.Y. Wu, and H.M. Han. Use of polymeric fullerene as a new coating for solid-phase microextraction. Chromatographia 52: 803-809 (2000).

10. C.H. Xiao, S.Q. Han, Z.Y. Wang, J. Xing, and C.Y. Wu. Application of the polysilicone fullerene coating for solid-phase microextraction in the determination of semi-volatile compounds. J. Chromatogr. A 927: 121-30 (2001).

11. J.Y. Yu, L. Dong, C.Y. Wu, L. Wu, and J. Xing. Hydroxyfullerene as a novel coating for solid phase micrextraction fiber with sol-gel technology. J. Chromatogr. A 978: 37-48 (2002).

12. Z.R. Zeng, W.L. Qiu, and Z.F. Huang. Solid-phase microextraction using fused-silica fibers coated with sol-gel-derived hydroxy-crown ether. Anal. Chem. 73: 2429-36 (2001).

13. Y. Lei. High extraction effect solid phase microextraction fibers coated with open crown ether stationary phase using sol-gel technique. Anal. Chim. Acta 486: 63-72 (2003).

14. Z.R. Zeng, W.L. Qiu, M. Yang, X. Wei, Z.F. Huang, and F. Li. Solidphase microextraction of monocyclic aromatic amines using novel fibers coated with crown ether. J. Chromatogr. A 934: 51-57 (2001).

15. D.H. Wang, J. Xing, J.G. Peng, and C.Y. Wu. Novel benzo-15crown-5 sol-gel coating for solid phase microextraction. J. Chromatogr. A 1005: 1-12 (2003).

16. J.X. Yu, C.Y. Wu, and J. Xing. Development of new solid phase microextraction fibers by sol-gel technology for the determination of organophosphorus pesticide multiresidures in food. J. Chromatogr. A 1036: 101-11 (2004).

17. L.S. Cai, Y.Y. Zhao, S.L. Gong, L. Dong, and C.Y. Wu. Use of a novel sol-gel dibenzo-18-crown-6 solid phase microextraction fiber coupling with new derivatizing reagent for determination of aliphatic amines in lake water and human urine. Chromatographia 58: 1-7 (2003).

18. M. Yang, Z.R. Zeng, W.L. Qiu, and Z.F. Huang. Preparation and investigation of a solid-phase microextraction fiber coated with open chain crown ether containing vinyl group. Chem. J. Chin. Univ. 24: 615-17 (2003).

19. X.J. Li, Z.R. Zeng, S.Z. Gao, and H.B. Li. Preparation and characteristics of sol-gel-coated calix[4]arene fiber for solid phase microextraction. J. Chromatogr. A 1023: 15-25 (2004).

20. X.J. Li, Z.R. Zeng, J.J. Zhou, S.L. Gong, W. Wang, and Y.Y. Chen. Novel fiber coated with amide bridge-calix[4]arene used for solid phase microextraction of aliphatic amines. J. Chromatogr. A 1041: 1-9 (2004).

21. X.J. Li, Z.R. Zeng, and J.J. Zhou. High thermal-stable sol-gel-coated calix[4]arene fiber for solid phase microextraction of chlorophenols. Anal. Chim. Acta 509: 27-37 (2004).

22. Y.L. Hu, Y.X. Zheng, and G.K. Li. Solid phase microextraction of phenol compounds using a fused-silica fiber coated with $\beta$-cyclodextrin-bonded silica particles. Anal. Sci. 20: 667-71 (2004).

23. Y.L. Hu, Y.Y. Yang, J.X. Huang, and G.K. Li. Preparation and application of poly(dimethylsiloxane)/cyclodextrin solid-phase microextraction membrane. Anal. Chim. Acta 543: 17-24 (2005).

24. X.Z. Du, Y.R. Wang, X.J. Tao, and H.L. Deng. An approach to application of mesoporous hybrid as a fiber coating of solid-phase microextraction. Anal. Chim. Acta 543: 9-16 (2005).

25. Y. Fan, Y.Q. Feng, Z.G. Shi, and J.B. Wang. Ordered mesoporous silica coated capillary for in-tube solid phase microextraction coupled to high-performance liquid chromatography. Anal. Chim. Acta 543: 1-8 (2005).

26. T.H. Ding, H.H. Lin, and C.W. Whang. Determination of chromium(III) in water by solid-phase microextraction with a polyimide-coated fiber and gas chromatography-flame photometric detection. J. Chromatogr. A 1062: 49-55 (2005).

27. R.Q. Yang and W.L. Xie. Preparation and usage of a new solid phase micro-extraction membrane. Forensic Sci. Int. 139: 177-81 (2004).

28. M.A. Farajzadeh and M. Hatami. A new selective SPME fiber for some $n$-alkanes and its use for headspace sampling of aqueous samples. J. Sep. Sci. 26: 802-808 (2003).

29. P.G. Thompson, C. Ozcan, L.S. Karen, F.R. Judith, G. Ahmed, A.C. Joseph, and B.M. Harry. Electrochemical control of solid phase micro-extraction using unique conducting polymer coated fibers. Anal. Com. 36: 203-205 (1999).

30. T. Ugur, Y. Brian, G. Ahmed, G. Thompson, L. Rebecca, S. Carla, T. Khalid, C. Özcan, and B.M. Harry. Electrochemically aided control of solid phase micro-extraction (EASPME) using conducting polymer-coated solid substrates applicable to neutral analytes. Mikrochim. Acta 143: 205-15 (2003).

31. G. Liljegren, J. Pettersson, K.E. Markides, and L. Nyholm. Electrochemical solid phase microextraction of anions and cations using polypyrrole coatings and an intergrated three-electrode device. Analyst 127: 591-97 (2002).

32. T. Ugus, S. Yücel, E. Nusret, U. Yasemin, P. Kadir, and Y. Attila. Preparation of sulfonated overoxidized polypyrrole film applicable as an SPME tool for cationic analytes. J. Electroanal. Chem. 570: 6-12 (2004).

33. U. Tamer, N. Ertas, Y.A. Udum, Y. Sahin, K. Pekmez, and A. Yildiz. Electrochemically controlled solid-phase microextraction based on overoxidized sulfonated polypyrrole. Talanta 67: 245-51 (2005).

34. H. Bagheri, A. Mir, and E. Babanezhad. An electropolymerized aniline-based fiber coating for solid phase microextraction of phenols from water. Anal. Chim. Acta 532: 89-95 (2005).

35. J.C. Wu, W.M. Mullett, and J. Pawliszyn. Electrochemically con- 
trolled solid phase microextraction based on conductive polypyrrole films. Anal. Chem. 74: 4855-59 (2002).

36. J.C. Wu and J. Pawliszyn. Preparation and applications of polypyrrole films in solid-phase microextraction. J. Chromatogr. A 909: 37-52 (2001).

37. J.C. Wu, H.L. Lord, J. Pawliszyn, and H. Kataoka. Polypyrrolecoated capillary in-tube solid phase microextraction coupled with liquid chromatography-electrospray ionization mass spectrometry for the determination of $\beta$-blockers in urine and serum samples. J. Microcol. Sep. 12: 225-66 (2000).

38. J. Wu, X. Yu, H. Lord, and J. Pawliszyn. Solid phase microextraction of inorganic anions based on polypyrrole film. Analyst 125: 391-94 (2000).

39. J.C. Wu, W. Xie, and J. Pawliszyn. Automated in-tube solid phase microextraction coupled with HPLC-ES-MS for the determination of catechins and caffeine in tea. Analyst 125: 2216-22 (2000).

40. J.C. Wu, C. Tragas, H. Lord, and J. Pawliszyn. Analysis of polar pesticides in water and wine samples by automated in-tube solid-phase microextraction coupled with high-performance liquid chromatography-mass spectrometry. J. Chromatogr. A 976: 357-67 (2002).

41. J.C. Wu, Z. Mester, and J. Pawliszyn. Determination of tributyltin by automated in-tube solid-phase microextraction coupled with HPLC-ES-MS. J. Anal. Atom. Spectrom. 16: 159-65 (2001).

42. J.C. Wu, Z. Mester, and J. Pawliszyn. Speciation of organoarsenic compounds by polypyrrole-coated capillary in-tube solid phase microextraction coupled with liquid chromatography/electrospray ionization mass spectrometry. Anal. Chim. Acta 424: 211-22 (2000).

43. J.C. Wu and J. Pawliszyn. Solid-phase microextraction based on polypyrrole films with different counter ions. Anal. Chim. Acta 520: 257-64 (2004).

44. J. Wu and J. Pawliszyn. Polypyrrole-coated capillary controlled solid phase microextraction based on conductive polypyrrole films. Anal. Chem. 73: 55-63 (2001).

45. Y. Wang, M. Walles, B. Thomson, S. Nacson, and J. Pawliszyn. Solid phase microextraction combined with surface enhanced laser desorption/ionization introduction for ion mobility spectrometry and mass spectrometry using polypyrrole coatings. Rapid Comm. Mass Spectrom. 18: 157-62 (2004).

46. Y. Mohammdi, Y Yamini, and N. Alizadeh. Dodecylsulfate-doped polypyrrole film prepared by electrochemical fiber coating technique for headspace solid phase microextraction of polycyclic aromatic hydrocarbons. J. Chromatogr. A 1063: 1-8 (2005).

47. H. Lord, R.P. Grant, M. Walles, B. Incledon, B. Fahie, and J. Pawliszyn. Development and evaluation of a solid phase microextraction probe for in vivo pharmacyokinetic studies. Anal. Chem. 75: 5103-15 (2003).

48. E.H.M. Koster, C. Crescenzi, W. Hoedt, K. Ensing, and G.J. de Jong. Fibers coated with molecularly imprinted polymers for solid-phase microextraction. Anal. Chem. 73: 3140-45 (2001).

49. W.M. Mullett, P. Martin, and J. Pawliszyn. In-tube molecularly imprinted polymer solid-phase microextraction for the selective determination of propranolol. Anal. Chem. 73: 2383-89 (2001).

50. F. Mangani and R. Cenciarini. Solid phase microextraction using fused silica fibers coated with graphitized carbon black. Chromatographia 41: 678-84 (1995).

51. D. Djozan and Y. Assadi. A new porous layer activated-charcoalcoated fused silica fiber: application for determination of BTEX compounds in water sample using headspace solid-phase microextraction and capillary gas chromatography. Chromatographia 45: 183-89 (1997).

52. D. Djozan and Y. Assadi. Modified pencil lead as a new fiber for solid-phase microextraction. Chromatographia 60: 313-17 (2004).

53. T. Sun, J.P. Jia, N. Fang, and Y. Wang. Application of novel activated carbon fiber solid-phase microextraction to the analysis of chlorinated hydrocarbons in water by gas chromatography-mass spectrometry. Anal. Chim. Acta 530: 33-40 (2005).

54. R. Aranda, P. Kruus, and R.C. Burk. Assessment of polycrystalline graphites as sorbents for solid phase microextraction of nonionic surfactants. J. Chromatogr. A 888: 35-41 (2000).

55. M. Giardina and S.V. Olesik. Application of low temperature glassy carbon films in solid phase microextraction. Anal. Chem. 73: 5841-51 (2001).

56. M. Ciardina and S.V. Olesik. Application of low-temperature glassy carbon-coated macrofibers for solid-phase microextraction analysis of simulated breath volatiles. Anal. Chem. 75: 1604-14 (2003).

57. M. Giardina, L.H. Ding, and S.V. Olesik. Development of fluorinated low temperature glassy carbon films for solid-phase microextraction. J. Chromatogr. A 1060: 215-24 (2004).

58. D. Djozan, Y. Assadi, and G. Karim-Nezhad. Modified copper wire as solid-phase microextraction fiber selective extraction of some amines. Chromatographia 56: 611-16 (2002).

59. D. Djozan, Y. Assadi, and S.H. Haddadi. Anodized aluminum wire as a solid phase microextraction fiber. Anal. Chem. 73: 4054-58 (2001).

60. D. Djozan and L. Abdollahi. Anodized zinc wire as a solid phase microextraction fiber. Chromatographia 57: 799-804 (2003).

61. D. Djozan and M. Amir-Zehmi. Copper sulfide wire as a selective fiber in solid phase microextraction. Chromatographia 58: 221-24 (2003).

62. M.A. Farajzadeh and N.A. Rahmani. Electrolytically produced copper(I) chloride on the copper wire as an excellent sorbent for some amines. Talanta 65: 700-704 (2005).

63. M.A. Farajzadeh and N.A. Rahmani. Alumina-based fiber for solid phase microextraction of alcohols from gaseous samples. Anal. Sci. 20: 1359-62 (2004).

64. L.M. Wei, Q.Y. Ou, and J.B. Li. Preparation of a solid phase microextraction fiber coated with gamma- $\mathrm{Al}_{2} \mathrm{O}_{3}$ and determination of volatile organic compounds in indoor air. Chromatographia 59: 601-605 (2004).

65. F. de Oliveira, C.B. da Sileira, S.D. De Campos, E.A. De Campos, and E. Carasek. Niobium (V) oxide coated on thin glass-ceramic rod as a solid phase microextracion fiber. Talanta 66: 74-79 (2005).

66. B. da Silveira, A.F. De Oliveira, S.D. De Campos, É.A. De Campos, and E. Carasek. Glass fibers coated with $\mathrm{Nb}_{2} \mathrm{O}_{5}$ for use in SPME. Colloids Surfaces A: Physicochem. Eng. Aspects 259: 15-22 (2005).

67. J. Pawliszyn and V. Mani. Applications of Solid Phase Microextraction, 1st ed. J. Pawliszyn, Ed. The Royal Society of Chemistry, Corwall, U.K., 1999, pp. 51-72.

68. S.L. Chong, D.X. Wang, and J.D. Hayes. Sol-gel coating technology for the preparation of solid-phase microextraction fibers of enhanced thermal stability. Anal. Chem. 69: 3889-98 (1997).

69. M.M. Liu, Z.R. Zeng, and Y. Tian. Elimination of matrix effects for headspace solid-phase microextraction of important volatile compounds in red wine using a novel coating. Anal. Chim. Acta 540: 341-53 (2005).

70. M.M. Liu, Z.R. Zeng, and H.F. Fang. Preparation and application of the sol-gel-derived acrylate/silicone co-polymer coatings for headspace solid-phase microextraction of 2-chloroethyl ethyl sulfide in soil. J. Chromatogr. A 1076: 16-26 (2005).

71. F.R. Zhou, X.J. Li, and Z.R. Zeng. Determination of phenolic compounds in wastewater samples using a novel fiber by solid-phase microextraction coupled to gas chromatography. Anal. Chim. Acta 538: 63-70 (2005).

72. C.Z. Dong, Z.R. Zeng, and X.J. Li. Determination of organochlorine pesticides and their metabolites in radish after headspace solidphase microextraction using calix[4] arene fiber. Talanta 66: 721-27 (2005).

73. M.M. Liu, Z.R. Zeng, and B. Xiong. Preparation of novel solid-phase microextraction fibers by sol-gel technology for headspace solidphase microextraction-gas chromatographic analysis of aroma compounds in beer. J. Chromatogr. A 1065: 287-99 (2005).

74. M. Ligor, M. Scibiorek, and B. Buszewski. New coating surfaces of fibers for solid-phase microextraction. J. Micro. Sep. 5: 377-83 (1999).

75. T.P. Gbatu, K.L. Sutton, and J.A. Caruso. Development of new SPME fibers by sol-gel technology for SPME-HPLC determination of organometals. Anal. Chim. Acta 402: 67-79 (1999). 
76. K. Alhooshani, T.Y. Kim, A. Kabir, and A. Malik. Sol-gel approach to in situ creation of high $\mathrm{pH}$-resistant surface-bonded organic-inorganic hybrid zirconia coating for capillary microextraction (in-tube SPME). J. Chromatogr. A 1062: 1-14 (2005).

77. C. Basheer, S. Jegadesan, S. Valiyaveettil, and H.K. Lee. Sol-gelcoated oligomers as novel stationary phases for solid-phase microextraction. J. Chromatogr. A 1087: 252-58 (2005).

78. R.G. da C. Silva and F. Augusto. Highly porous solid-phase microextraction fiber coating based on poly(ethylene glycol)-modified ormosils synthesized by sol-gel technology. J. Chromatogr. A 1072: 7-12 (2005).

79. H. Bagheri, A. Es-haghi, and M.R. Rouini. Sol-gel-based solidphase microextraction and gas chromatography-mass spectrometry determination of dextromethorphan and dextrorphan in human plasma. J. Chromatogr. B 818: 147-57 (2005).

80. M. Azenha, C. Malheiro, and A.F. Silva. Ultrathin phenyl-functionalized solid phase microextraction fiber coating developed by sol-gel deposition. J. Chromatogr. A 1069: 163-72 (2005).

81. T. Górecki, P. Martos, and J. Pawliszyn. Strategies for the analysis of polar solvents in liquid matrixes. Anal. Chem. 70: 19-27 (1998).

82. J.P. Jia and Y. He. Preparation of new coating for solid phase microextraction. Chem. World 4: 214-15 (1998).

83. H. Bin, G.B. Jiang, and Z.M. Ni. Determination of methylmercury in biological samples and sediments by capillary gas chromatography coupled with atomic absorption spectrometry after hydride derivatization and solid phase microextraction. J. Anal. Atom. Spectrom. 13: 1141-44 (1998).

84. Y. Liu, Y. Shen, and M.L. Lee. Porous layer solid-phase microextraction using silica bonded phases. Anal. Chem. 69: 190-95 (1997).

85. Y. Liu, M.L. Lee, K.J. Hageman, Y. Yang, and S.B. Hawthorne. Solidphase microextraction of $\mathrm{PAHs}$ from aqueous samples using fibers coated with HPLC chemically bonded silica stationary phases. Anal. Chem. 69: 5001-5005 (1997).

86. M. Walles, W.M. Mullett, and J. Pawliszyn. Monitoring of drugs and metabolites in whole blood by restricted-access solid-phase microextraction coupled to liquid chromatography-mass spectrometry. J. Chromatogr. A 1025: 85-92 (2004).

87. F.M. Musteata, M. Walles, and J. Pawliszyn. Fast assay of angiotensin 1 from whole blood by cation-exchange restrictedaccess solid-phase microextraction. Anal. Chim. Acta 537: 231-37 (2005).

88. J.G. Hou, Q. Ma, X.Z. Du, H.L. Deng, and J.Z. Gao. Inorganic/ organic mesoporous silica as a novel fiber coating of solid-phase microextraction. Talanta 62: 241-46 (2004).

89. M. Trojanowicz. Application of conducting polymers in chemical analysis. Microchim. Acta 143: 75-91 (2003).

90. H. Bagheri, A. Mohammadi, and A. Salemi. On-line trace enrichment of phenolic compounds from water using a pyrrole-based polymer as the solid-phase extraction sorbent coupled with highperformance liquid chromatography. Anal. Chim. Acta 513: 445-49 (2004).

91. H. Bagheri and A. Mohammadi. Pyrrole-based conductive polymer as the solid phase extraction medium for the preconcentration of environmental poluttants in water samples followed by gas chromatography with flame ionization and mass spectrometry detection. J. Chromatogr. A 1015: 23-20 (2003).

92. H. Ge and G.G. Wallac. Characterization of conducting polymeric stationary phases and electrochemically controlled high-performance liquid chromatography. Anal. Chem. 61: 2391-84 (1989).

93. C. Perruchot, M.M. Chehimi, M. Delamar, and F. Dardoize. Characterization of the chromatographic properties of a silicapolypyrrole composite stationary phase by inverse liquid Chromatography. J. Chromatogr. A 969: 167-80 (2002).

94. J.Y. Sung and H.J. Huang. Application of a polyaniline-Nafion composite electrode to the determination of alkali and alkaline earth metal ions using flow-injection analysis and ion chromatography. Anal. Chim. Acta 246: 275-81 (1991).

95. R.S. Deinhammer, K. Shimazu, and M.D. Porter. Ion chromatographic separation using step and linear voltage waveforms at a charge controllable polymeric stationary phase. Anal. Chem. 63: 1894-97 (1991).

96. M.J. Huang, C. Tai, Q.F. Zhou, and G.B. Jiang. Preparation of polyaniline coating on a stainless-steel wire using electroplating and its application to the determination of six aromatic amines using headspace solid-phase microextraction. J. Chromatogr. A 1048: 257-62 (2004).

97. M.J. Huang, G.B. Jiang, and Y.Q. Cai. Electrochemical preparation of composite polyaniline coating and its application in the determination of bisphenol A, 4-n-nonylphenol, 4-tert-octylphenol using direct solid phase microextraction coupled with high performance liquid chromatography. J. Sep. Sci. 28: 2218-24 (2005).

98. A. Ghassempour, N.M. Najafi, A. Mehdinia, S. Saeed, H. Davarani, M. Fallahi, and M. Nakhshab. Analysis of anatoxin-a using polyaniline as a sorbent in solid-phase microextraction coupled to gas chromatography-mass spectrometry. J. Chromatogr. A 1078: 120-27 (2005).

99. W.M. Mullett and J. Pawliszyn. The development of selective and biocompatible coating for solid phase microextraction. J. Sep. Sci. 26: 251-60 (2003).

100. H.D. Yuan, W.M. Mullett, and J. Pawliszyn. Biological sample analysis with immunoaffinity solid-phase microextraction. Analyst 126: 1456-61 (2001).

101. Y. Fan, Y.Q. Feng, J.T. Zhang, S.L. Da, and M. Zhang. Poly(methacrylic acid-ethylene glycol dimethacrylate) monolith intube solid phase microextraction coupled to high performance liquid chromatography and analysis of amphetamines in urine samples. J. Chromatogr. A 1074: 9-16 (2005).

102. Y. Fan, Y.Q. Feng, S.L. Da, and Z.G. Shi. Poly(methacrylic acidethylene glycol dimethacrylate) monolithic capillary for in-tube solid phase microextraction coupled to high performance liquid chromatography and its application to determination of basic drugs in human serum. Anal. Chim. Acta 523: 251-58 (2004).

Manuscript received September 14, 2005; revision received January 26, 2006. 